\title{
SIMULAÇÃO DO ZONEAMENTO ECOLÓGICO DA LAGARTA-DO-CARTUCHO NO RIO GRANDE DO SUL COM O AUMENTO DE TEMPERATURA
}

\author{
A.P.S. Afonso ${ }^{1}$, M. Wrege ${ }^{2}$, J.F. da S. Martins ${ }^{1}$, D.E. Nava ${ }^{1}$ \\ 1Embrapa Clima Temperado, CP 403,CEP 96001-970, Pelotas, RS, Brasil. E-mail: anapaula@cpact.embrapa.br
}

\section{RESUMO}

\begin{abstract}
A lagarta-militar é uma praga polífaga que causa grandes prejuízos às culturas do arroz e milho no Rio Grande do Sul. Vários estudos têm sido direcionados à minimização dos danos causados por ela. A constatação de biótipos de Spodoptera frugiperda no Rio Grande do Sul, associada a elevação da temperatura, criou novos cenários e implicações no manejo dessa praga. O objetivo desse trabalho foi definir o zoneamento ecológico de $S$. frugiperda para os biótipos "arroz" e "milho" no Estado, com base nas exigências térmicas do inseto para estimar o número de gerações, conforme o aumento das temperaturas mínimas, previsto pelo IPCC. Nas condições ecológicas atuais, o biótipo "arroz" pode atingir 5 a 9 gerações ao ano, porém, com aumentos de $1^{\circ}, 3^{\circ}$ e $5,8^{\circ}$ C, atingir de 5 a 10, 6 a 12 e 9 a 14 gerações, respectivamente. O biótipo "milho" atualmente pode atingir de 2 a 8 gerações ao ano, com os referidos aumentos de temperatura atingiria três a 8,6 a 10 e 8 a 13 gerações, respectivamente.
\end{abstract}

PALAVRAS-CHAVE: Spodoptera frugiperda, temperatura, distribuição de pragas.

\section{ABSTRACT}

ECOLOGICALZONINGOFTHEFALLARMYWORMINTHESTATEOFRIOGRANDEDOSUL, BRAZIL. The fall armyworm is a polyphagous pest that causes major damage to rice and maize crops in the state of Rio Grande do Sul, Brazil. Various studies have been conducted to minimize the damage caused by the larvae. The observation of biotypes of $S$. frugiperda in the state of Rio Grande do Sul, associated to higher temperature, has created new scenarios and implications in the management of this pest. The objective of the present study was to define the ecological zoning of $S$. frugiperda biotypes for "rice" and "corn" in the state, based on thermal requirements of the insect in order to estimate the number of generations, according to the increase in the minimum temperatures predicted by the IPCC. In the current ecological conditions, the biotype "rice" may reach 5 to 9 generations per year, however, with increases of 1,3 and $5.8^{\circ} \mathrm{C}$, it could reach up to 5 to 10,6 to 12 and 9 to 14 generations, respectively. The biotype "maize," which today may reach 2 to 8 generations per year, with these increases in temperature would reach 3 to 8,6 to 10 and 8 to 13 generations, respectively.

KEY WORDS: Spodoptera frugiperda, temperature, pest distribution.

\section{INTRODUÇÃO}

As culturas do arroz e do milho são básicas à alimentação humana, constituindo-se em fontes de calorias para grande parte da população mundial, portanto, assumindo relevante papel sócio econômico(WeBSTER; GunNELL, 1992). No Brasil, de acordo com a CONAB (Companhia Nacional de Abastecimento, 2008), em 2006/2007, foram cultivados aproximadamente 3 milhões dehectares com arroze 15 milhões de hectares com milho.

Diversas espécies de insetos danificam as culturas do arroz e do milho desde a semeadura à fase de formação dos grãos (GRÜTZMACHER, 1994; MARTINS; BOtTON, 1996; GRÜTZMACHER et al., 2000a). Dentre as mais prejudiciais, destaca-se Spodoptera frugiperda (J.E.Smith, 1797) (Lepidoptera: Noctuidae), conhecida por lagarta-da-folha e lagarta-do-cartucho, nas culturas do arroz e do milho, respectivamente(VIANA et al., 2004).

Na cultura do arroz, no Rio Grande do Sul, principalmente em áreas planas, o ataque das lagartas de S. frugiperda émais crítico no período entre a emergência das plantas e a inundação da lavoura, quando corta os colmos de plantas novas rente ao solo. Em áreas inclinadas (lavouras de coxilha), o ataque se

${ }^{2}$ Embrapa Florestas, Colombo, PR, Brasil. 
estendeaplantasmais desenvolvidas, principalmenteàs localizadas sobre as taipas, englobando desfolhamentos e danos às flores e panículas (MARTINS, 2004).

Na cultura do milho, a capacidade de dano das lagartas de $S$. frugiperda é influenciada pelo vigor das plantas e pelo clima. As lagartas de primeiro e segundo instar consomem parte das folhas e mantém a epidermeintacta, aparentando sintoma de raspagem. Em instares mais avançados perfuram as folhas e estabelecem-se no cartucho do milho. Ainda, podem perfurar a base dos colmos e atacar as espigas. $\mathrm{O}$ ataqueémais crítico quando ocorre sobre plantas com 8 a 10 folhas, podendo causar redução de 19\% no rendimento (GRÜTZMACHER et al., 2000b).

Recentemente foi constatada a presença debiótipos "arroz" e "milho" de S. frugiperda, no Rio Grande do Sul (BusAto et al., 2004). Reações diferenciadas de biótipos, quanto à suscetibilidade a inseticidas, resistência de plantas e preferência para oviposição têm sido observadas (ADAMCZYKet al., 1997; EDWARDsetal., 1999). O biótipo "arroz" demostrou ser mais suscetível a inseticidas como o diazinom e carbaryl, e o biótipo "milho" ao carbofurano. Ademais, lagartas do biótipo "arroz" demonstraram ser mais suscetíveis a Bacillus thuringiensis.

Váriosestudostêmsidorealizadosvisandominimizar osdanoscausadospelalagarta, tantoemarrozquantoem milho.Umdosfatoresquedeveserconsideradorefere-se às mudanças na temperatura. Há pouco conhecimento sobre os efeitos dessas mudanças nos diversos ecossistemas brasileiros. Apesar de alguns fenômenos evidenciarem uma relaçãoentre as mudanças climáticas ealterações na biodiversidade animal e vegetal, as informações não são precisas. $\mathrm{O}$ aumento da temperatura média global poderá causar o declínio populacional de plantas e animais e já tem sido observado que algumas espéciesdeplantastêmflorescidomaiscedoe, alémdisso, já foi atribuída às mudanças climáticas a proliferação de certas espécies deinsetos, bem como, alterações na duraçãododesenvolvimento, tamanho,longevidade,fecundidade, razão sexual, comportamento e distribuição geográfica (HENCE et al., 2007).

As pesquisas sobre o efeito de mudanças climáticas na agricultura brasileira ainda são restritas (SIQUEIRA, 2001), no entanto, os estudos nesse sentido tornam-se cada vez mais importantes. Revisão bibliográfica das pesquisas desenvolvidas no mundo sobre este tema (GHINI, 2005) indica que as mudanças no clima poderão modificar a importância relativa de insetos-praga e doenças das principais culturas, com consequente alteração no atual cenário fitossanitário da agricultura brasileira.

Oobjetivo desse trabalho foi realizar o zoneamento ecológico de S. frugiperda biótipos "arroz" e "milho" para o Rio Grande doSul, baseando-senas exigências térmicas, esimular o aumento do número degerações conforme o aumento das temperaturas mínimas, previstas no relatório de mudanças climáticas globais pelo Painel Intergovernamental sobre Mudança Climática (IPCC, 2007).

\section{MATERIALEMÉTODOS}

Para calcular a soma térmica (graus-dia), foram usados dados climáticos de séries históricas da Fundação Estadual de Pesquisa Agropecuária do Rio Grande do Sul (Fepagro) e do $8^{\circ}$ Distrito de Meteorologia do Instituto Nacional de Meteorologia ( $8^{\circ}$ Disme/Inmet). Em relação às temperaturas máxima (Tmax) e mínima (Tmin) diárias, foi calculado o somatório de horas de calor (GD) superiores ao limiar térmico inferior de desenvolvimento ou temperatura base(Tbase) de 9,6 e 10,9 $9^{\circ}$, determinados por BUSATO et al.(2005), respectivamente, para os biótipos "arroz" e "milho" de S. frugiperda. A soma térmica foi calculada para todos os meses do ano, sendo utilizados como referencial os valores de 470,5 e 463,0 grausdias (GD) necessários para completar uma geração dos biótipos "arroz" e "milho", respectivamente (BusAto et al., 2005), segundo a seguinte fórmula:

$$
G D=[(\text { Tmáx }+T \min ) / 2]-\text { Tbase }
$$

Aos valores de Tmin foram adicionados $1^{\circ} \mathrm{C}, 3^{\circ} \mathrm{C}$ e $5,8^{\circ} \mathrm{C}$, simulando aquecimentos do ar com a elevação de temperaturas, previstos para ocorrerem até o final do século XXI, em consequência de mudanças climáticas globais decorrentes do aumento do $\mathrm{CO}_{2}$ na atmosfera (IPCC, 2007). Os novos valores de Tmin foram aplicados à equação GD, simulando cenários de aquecimento global de $1^{\circ} \mathrm{C}$, nos próximos 10 anos, e de $5,8^{\circ}$ C, nos próximos 90 anos. Os dados foram interpolados por meio de sistemas de informações geográficas, utilizando o programa ArcGIS, versão 9, de acordo com a altitude, latitudeelongitude. Usaram-se equações que relacionam o número de gerações da lagarta-do-cartucho com as coordenadas geográficas, obtidas a partir de séries históricas (Tabela 1).

Utilizaram-se valores de altitude do modelo digital de elevação do SRTM (U.S. GeOLOGICAL SURVEY, 1999), adaptado para o sistema brasileiro de referência oficial por WEBER et al. (2004), nos quais os pontos são distribuídos espacialmente comouma grade, com um valor a cada 90 metros. A latitude e a longitude foram determinadas da mesma forma (Tabela 1).

\section{RESULTADOSEDISCUSSÃO}

O biótipo "arroz" de S. frugiperda, detectado no Rio Grande doSul(BusATOet al., 2004), nas condições 
atuais de temperatura, pode atingir de 5 a 9 gerações ao ano (Fig. 1A). Omenor número de gerações ocorre na região Nordeste e no extremo Sul, e o maior número na região Noroeste do Estado, nas quais tradicionalmente o arroz não é cultivado. Na região do extremo Sul, produtora de arroz, o inseto atinge 6 gerações.

Considerando-se uma elevação de $1^{\circ} \mathrm{C}$ na temperatura mínima do ar (Fig. 1B), no geral, haveria o aumento de uma geração de $S$. frugiperda em quase todas as regiões. Nas regiões orizícolas como a Sul e a Fronteira Oesteesta elevação, possivelmente, incorreria em aumento do risco de danos à cultura. Para um aumento de $3^{\circ} \mathrm{C}$ na temperatura mínima do ar, o número de gerações ao ano oscilaria de 6a 12 (Fig.1C). Nessa circunstância, áreas tradicionais de produção de arroz seriam amplamente afetadas. Em caso de uma elevação de $5,8^{\circ} \mathrm{C}$ na temperatura, no RioGrande do Sul, o biótipo "arroz" de S. frugiperda atingiria de 9 a 14 gerações ao ano, independente da região orizícola, e os danos da lagarta-da-folha seriam consideráveis (Fig. 1D).
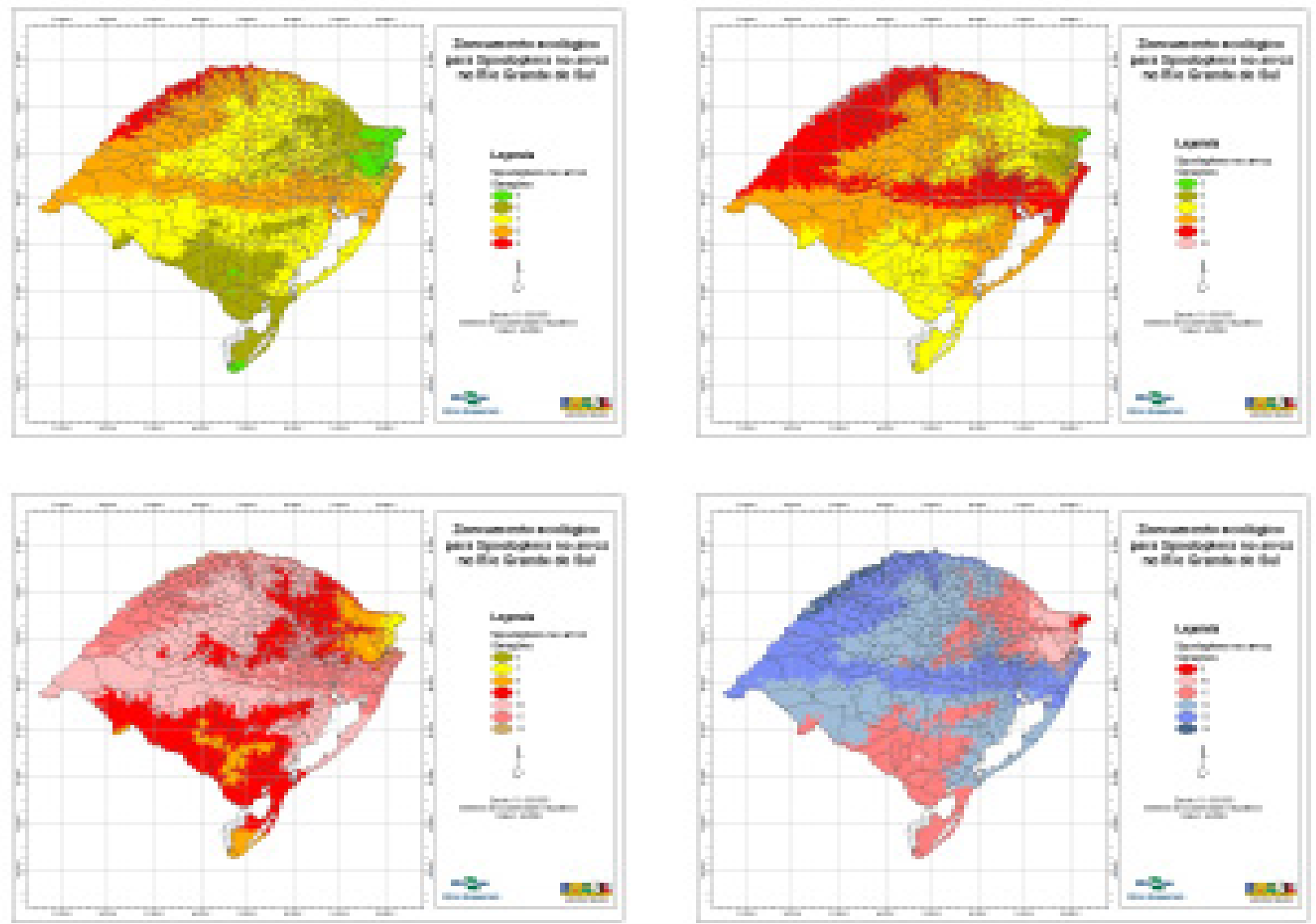

Fig. 1 - Previsão do número de gerações anuais do biótipo arroz de Spodoptera frugiperda em função das condições climáticas atuais do Rio Grande do Sul (A) e de aumentos de $1^{\circ} \mathrm{C}(\mathrm{B}), 3^{\circ} \mathrm{C}(\mathrm{C})$ e $5,8^{\circ} \mathrm{C}$ (D) na temperatura mínima do ar.

Tabela 1 - Equações utilizadas na previsão do número de gerações de Spodoptera frugiperda em função da altitude, latitude e longitude das estações meteorológicas do Rio Grande do Sul.

\begin{tabular}{llr}
\hline Temperatura mínima do ar $\left({ }^{\circ} \mathrm{C}\right)$ & Equação de regressão & $\mathrm{R}^{2}$ \\
\hline Atual & $25,31+0,651^{*}$ latitude- $0,030^{*}$ longitude- $0,0032^{*}$ altitude & 0,68 \\
$+1^{\circ} \mathrm{C}$ & $27,15+0,534^{*}$ latitude+0,05**longitude- $0,0034^{*}$ altitude & 0,55 \\
$+3^{\circ} \mathrm{C}$ & $17,97+0,486^{*}$ latitude-0,100*longitude- $0,0033^{*}$ altitude & 0,67 \\
$+5,8^{\circ} \mathrm{C}$ & $27,51+0,662^{*}$ latitude-0,038*longitude-0,0029*altitude & 0,47 \\
\hline
\end{tabular}



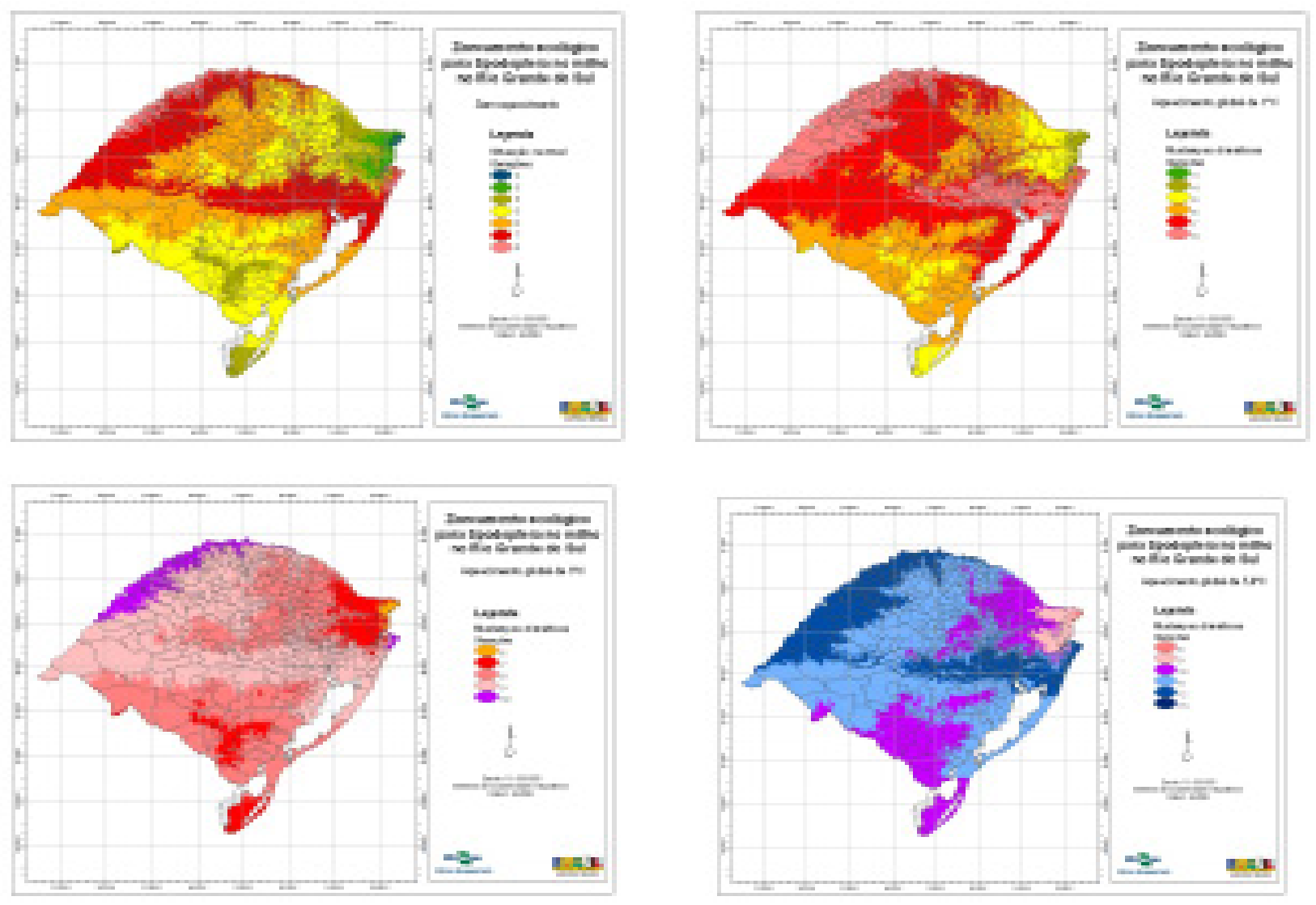

Fig. 2 - Previsão do número de gerações anuais do biótipo milho de Spodoptera frugiperda em função das condições climáticas atuais do Rio Grande do Sul (A) e de aumentos de $1^{\circ} \mathrm{C}(\mathrm{B}), 3^{\circ} \mathrm{C}(\mathrm{C})$ e 5,8 $\mathrm{C}$ (D) na temperatura mínima do ar.

Em relação ao biótipo "milho" de $S$. frugiperda detectadono Rio Grande doSul, nas condições atuais, seriam atingidas de 2 a 8 gerações ao ano (Fig. 2A). Menor número de gerações do biótipo (2 a 4), porém, ocorrena região Nordeste do Estado, provavelmente devido ao fato da região não se caracterizar como produtora de milho. Por outro lado, no Centro Nordeste, Fronteira Oeste e Sul, o biótipo desenvolve 5 a 6 gerações. Nas demais regiões ocorrem 7 gerações, até atingir 8 na região Noroeste, de maior concentração de municípios produtores de milho. Havendo uma elevação na temperatura mínima do ar de $1^{\circ} \mathrm{C}$ (Fig. 2B), o cenário seria completamente diferente. Em áreas onde ocorriam somente 2 gerações, seriam detectadas de 3 a 5, sendo que no restante do Estado ocorreriam de 6 a 8 gerações. Um aumento de $3^{\circ} \mathrm{C}$ na temperatura mínima do ar implicaria em mudanças ainda mais significativas. Apenas no extremo da região Nordeste, onde atualmente ocorrem 2 gerações, poderiam surgir 6 gerações, sendo essa a região menos favorável ao biótipo. Nas demais regiões, ocorreriam de 7 a 9 geraçõese, no Noroeste, mais propício ao desenvolvimento do biótipo, até mesmo 10 gerações (Fig. 2C). Nocaso deum aumento da temperatura mínima do ar para $5,8^{\circ} \mathrm{C}$, a situação se tornaria altamentedesfavorávelà cultura do milho, mesmono extremo Nordeste do Estado, onde ocorreriam de 8 a 9 gerações enquanto no restante do Estado, de 10 a 13 (Fig. 2D).

Os resultados obtidos neste trabalho, sobre simulação de aquecimento global, com elevação da temperatura, sem considerar efeitos da disponibilidade de alimento e deinimigos naturais, no desenvolvimento dos biótipos "arroz" e "milho" de S. frugiperda, indicaram que determinadas regiões do Rio Grande do Sul tornariam-se impróprias ao cultivo de ambos os cereais. A lagarta-da-folha ou lagarta-do-cartucho sendo polífaga encontra alimento ao longo do ano. Assim, se fatores climáticos ainda favorecerem seu desenvolvimento, a tendência é haver dificuldade crescente para o cultivo de arroz e milho em determinadas regiões. Outro fator agravante é que a existência de biótipos de $S$. frugiperda não condiciona o ataque a uma ou outra cultura, o que existe são insetos mais adaptados ao arroz ou ao milho (BusATo et al., 2004), o que não impede que cada biótipo ataque a outra cultura. Estes fatos reforçam a necessidade de estudos de zoneamento ecológico, que poderão defi- 
nir o grau de risco de ataque da lagarta nas diferentes regiões produtoras de arroz e milho. No entanto, maior importância deve ser direcionada à segunda safra (safrinha) de milho, visto as condições ecológicas da época (temperaturas elevadas e período mais propicio a estiagem) serem mais favoráveis ao desenvolvimento do inseto.

\section{CONCLUSÕES}

1. Obiótipo "arroz" de S. frugiperda podeatingir de 5 a 9 gerações ao ano nas condições térmicas atuais do estado do Rio Grande do Sul, e 5 a 10, 6 a 12, e 9 a 14 gerações por ano, com aumentos de $1^{\circ} \mathrm{C}, 3^{\circ} \mathrm{Ce} 5,8^{\circ} \mathrm{C}$, respectivamente.

2. Obiótipo "milho" de S. frugiperda pode atingir de 2 a 8 gerações ao ano nas condições térmicas atuais do estado do Rio Grande do Sul, e 3 a 8, 6 a 10 e 8 a 13 gerações ao ano, com aumentos de $1^{\circ} \mathrm{C}, 3^{\circ} \mathrm{C}$ e $5,8^{\circ} \mathrm{C}$, respectivamente, para o Estado do Rio Grande do Sul.

\section{REFERÊNCIAS}

ADAMCZYK, J.J.; HOLLOWAY, J.W.; LEONARD, B.R.; GRAVES, J. B. Susceptibility of fall armyworm collected from different plant hosts to selected insecticides and trangenic Bt cotton. Journal of Cotton Science, v.1, p.21-28, 1997.

BUSATO, G.R.; GRÜTZMACHER, A.D.; OLIVEIRA, A.C.; VIEIRA, E.A.; ZIMMER, P.D.; KOPP, M.M.; BANDEIRA, J.M.; MAGALHÃES, T. Análise da estrutura e diversidade molecular de populações de Spodoptera frugiperda (J.E. Smith) (Lepidoptera: Noctuidae) associadas às culturas de milho e arroz no Rio Grande do Sul. Neotropical Entomology, v.33, p.709-716, 2004.

BUSATO, G.R.; GRÜTZMACHER, A.D.; GARCIA, M.S.; GIOLO, F.P.; ZOTTI, M.J.; BANDEIRA, J.M. Exigências térmicas e estimativa do número de gerações dos biótipos "milho" e "arroz" de Spodoptera frugiperda. Pesquisa Agropecuária Brasileira, v.40, p.329-335, 2005.

\section{COMPANHIA NACIONAL DE ABASTECIMENTO}

(Brasil).. Acompanhamento da safra brasileira de grãos. Disponível em: <http://www.conab.gov.br/ conabweb/download/safra/estudo_safra.pdf >. Acesso em: 15 jun. 2008.

EDWARDS, M.L.; MENDOZA, J.L.H.; RUBIO, A.P.; MOLINA-OCHOA, J.; LEZAMA-GUTIÉRREZ,R.; HAMM, J. J.; WISEMAN, B. R. Biological differences between five populations of fall armyworm (Lepidoptera: Noctuidae) collected from corn in Mexico. Florida Entomologist, v.82, p.254-262, 1999.
GHINI, R. (Ed.). Mudanças climáticas globais e doenças de plantas. Jaguariúna: Embrapa Meio Ambiente, 2005. 104p.

GRÜTZMACHER, A.D. Artrópodes associados a cultura do arroz irrigado em vários sistemas de cultivos. 1994. 100p. Dissertação (Mestrado) - Escola Superior de Agricultura "Luiz de Queiroz", Universidade de São Paulo, Piracicaba. 1994.

GRÜTZMACHER, A.D.; MARTINS, J.F.S.; CUNHA, U.S. Insetos-praga das culturas do milho e sorgo no agroecossistema de várzea. In: PARFitT, J.M.B.(Ed.) Produção de milho e sorgo na várzea. Pelotas: Embrapa Clima Temperado, 2000a. p.87-101. (Documentos, 74).

GRÜTZMACHER, A. D; NAKANO, O.; MARTINS, J. F. DA S.; GRÜTZMACHER, D. D.;LOECK, A. E. Danos da lagarta-da-folha, Spodoptera frugiperda no arroz irrigado em diferentes condições hídricas. Agropecuária Clima Temperado (Pelotas, v.3, n.2, p.151-163, 2000b.

HENCE, T.; BAAREN, J.; VERNON, P.; BOIVIN, G. Impact of extreme temperatures on parasitoids in a climate change perspective. Annual Review of Entomology, v.52, p.107-126, 2007.

IPCC - Intergovernmental Panel Climate Change. Climate Change 2007: The Physical Science Bases. Switzerland: IPCC, 2007. 18p. [Working group I Contribuition to the Fourth Assessment Report of the IPCC].

MARTINS, J.F. da S. Descrição e manejo integrado de insetos-praga em arroz irrigado. In: GOMES, A. da S.; MAGALHÃES, JÚNIOR, A.M. (Ed.). Arroz irrigado no Sul do Brasil. Pelotas: Embrapa Clima Temperado; Brasília, DF: Embrapa Informação Tecnológica, 2004. 899p. MARTINS, J.F. da S.; BOTTON, M. Controle de insetos da cultura do arroz irrigado. In: PESKE, S.T.; NEDEL, J.L.; BARROS, A.C.S. A. (Ed.) Produção de arroz. Pelotas: UFPel, 1996. Cap.7, p.277-304.

SIQUEIRA, O.J.F. de Efeitos potenciais das mudanças climáticas na agricultura brasileira e estratégias adaptativas para algumas culturas. In: LIMA, M.A. de; CABRAL, O.M.R.; MIGUEZ, J.D.G. (Ed.). Mudanças climáticas globais e a agropecuária brasileira. Jaguariúna: Embrapa Meio ambiente, 2001. p.33-63.

U.S.Geological Survey. Survey National Mapping Division: Global 30 Arc Second Elevation Data: Disponível em: <http:/ / edcwww.cr.usgs.gov/landdaac/ gtopo30>. Acesso em: 10 jul. 1999.

VIANA, P.A.; WAQUIL, J.M.; VALICENTE, F.H.; CRUZ, I. Ocorrência e controle de pragas na safrinha de milho nas regiões Norte e Oeste do Paraná. Sete Lagoas: Embrapa Milho e Sorgo, 2004. 8p. (Circular Técnica, 45). 
WEBER, E.; HASENACK, H.; FERREIRA, C.J.S. Adaptação do modelo digital de elevação do SRTM para o sistema de referência oficial brasileiro e recorte por unidade da federação. Porto Alegre: UFRGS Centro de Ecologia. Disponível em: <http://ecologia.ufrgs/ labgeo>. 2004. Acesso em: 10 jul. 2004.
WEBSTER, R.K.; GUNNELL, P.S. Compendium of rice diseases. St. Paul : The American Phytopathological Society, 1992. 62p.

Recebido em 23/9/08

Aceito em 5/10/09 